\title{
Coordinated Dynamic Spectrum Management of LTE-U and Wi-Fi Networks
}

\author{
Shweta Sagari*, Samuel Baysting*, Dola Saha ${ }^{\dagger}$, Ivan Seskar*, Wade Trappe*, Dipankar Raychaudhuri* \\ ${ }^{*}$ WINLAB, Rutgers University, \{shsagari, sbaysting, seskar, trappe, ray\}@ winlab.rutgers.edu \\ †NEC Labs America,dola@nec-labs.com
}

\begin{abstract}
This paper investigates the co-existence of Wi-Fi and LTE in emerging unlicensed frequency bands which are intended to accommodate multiple radio access technologies. WiFi and LTE are the two most prominent access technologies being deployed today, motivating further study of the inter-system interference arising in such shared spectrum scenarios as well as possible techniques for enabling improved co-existence. An analytical model for evaluating the baseline performance of coexisting Wi-Fi and LTE is developed and used to obtain baseline performance measures. The results show that both $\mathrm{Wi}-\mathrm{Fi}$ and LTE networks cause significant interference to each other and that the degradation is dependent on a number of factors such as power levels and physical topology. The model-based results are partially validated via experimental evaluations using USRP based SDR platforms on the ORBIT testbed. Further, internetwork coordination with logically centralized radio resource management across Wi-Fi and LTE systems is proposed as a possible solution for improved co-existence. Numerical results are presented showing significant gains in both Wi-Fi and LTE performance with the proposed inter-network coordination approach.
\end{abstract}

\section{INTRODUCTION}

Exponential growth in mobile data usage is driven by the fact that Internet applications of all kinds are rapidly migrating from wired PCs to mobile smartphones, tablets, mobile APs and other portable devices [1]. Industry has already started gearing up for the 1000x increase in data capacity, which has given rise to the concept of the 5 th Generation $(5 \mathrm{G})$ mobile network. The $5 \mathrm{G}$ vision, though, is not limited to matching the increase in mobile data demand, but it also includes an improved overall service-oriented user experience with immersive applications, such as high definition video streaming, real-time interactive games, applications in wearable mobile devices, ubiquitous health care, mobile cloud, etc. [2]-[4]. For such applications, the system needs to provide improved Quality of Experience (QoE), which can be factored in different ways: better cell/edge coverage (availability of service), lower latency (round trip time), lower power consumption (longer battery life), reliable services, cost-effective network, and support for mobility.

To meet such a high Quality-of-Service and system capacity demand, there have been three main solutions proposed [5]: a) addition of more radio spectrum for mobile services (increase in $\mathrm{MHz}$ ), b) deployment of small cells (increase in bits $/ \mathrm{Hz} / \mathrm{km}^{2}$ ), and c) efficient spectrum utilization (increase in bits/second $/ \mathrm{Hz} / \mathrm{km}^{2}$ ). Several spectrum bands, as shown in figure 11, have been opened up for mobile and fixed wireless broadband services. These include 2.4 and $5 \mathrm{GHz}$ unlicensed bands for the proposed unlicensed LTE operation

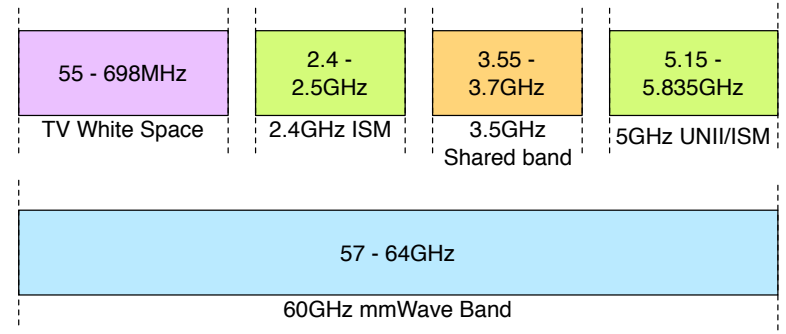

Fig. 1. Proposed spectrum bands for deployment of LTE/Wi-Fi small cells.

as a secondary LTE carrier [6]. These bands are currently utilized by unlicensed technologies such Wi-Fi/Bluetooth. The $3.5 \mathrm{GHz}$ band, which is currently utilized for military and satellite operations has also been proposed for small cell (WiFi/LTE based) services. Another possibility is the $60 \mathrm{GHz}$ band (millimeter wave technology), which is well suited for shortdistance communications including Gbps Wi-Fi, 5G cellular and peer-to-peer communications [7]. In addition, opportunistic spectrum access is also possible in TV white spaces for small cell/backhaul operations as secondary users [8].

These emerging unlicensed band scenarios will lead to co-channel deployment of multiple radio access technologies (RATs) by multiple operators. These different RATs, designed for specific purposes at different frequencies, now must coexist in the same frequency, time and space. This causes increased interference to each other and degradation of the overall system performance is eminent due to the lack of inter-RAT compatibility. Figure 2 shows two such scenarios, where the two networks interfere with each other. When Wi-Fi Access Point is within the transmission zone of LTE, it senses the medium and postpones transmission due to detection of LTE Home eNodeB's (HeNB) transmission power in the spectrum band as shown in figure 2(a) Consequently, the Wi-Fi link from AP to Client suffers in presence of LTE transmission. The main reason for this disproportionate share of the medium is due to the fact that LTE does not sense other transmissions before transmitting. On the other hand, Wi-Fi is designed to coexist with other networks as it senses the channel before any transmission. However, if LTE works as supplemental downlink only mode, UEs do not transmit at all. So, a WiFi AP, which cannot sense LTE HeNB's transmission, will transmit and cause interference at the nearby UEs, as shown in figure 2(b). This problem also exists in multiple Wi-Fi links with some overlap in collision domain, but the network can recover packets quickly as a) packets are transmitted for a very 


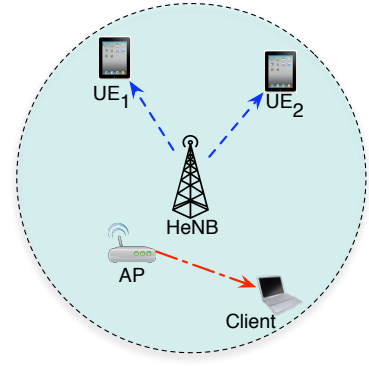

(a) Interference caused by LTE.

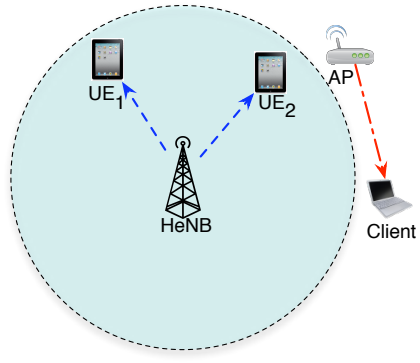

(b) Interference caused by Wi-Fi.
Fig. 2. Scenarios showing challenges of coexistence of LTE and Wi-Fi in the same unlicensed spectrum.

short duration in Wi-Fi, compared to longer frames in LTE and b) all the nodes perform carrier sensing before transmission. Therefore, to fully utilize the benefits of new spectrum bands and deployments of HetNets, efficient spectrum utilization needs to be provided by the dynamic spectrum coordination framework and the supporting network architecture.

It is reasonable to forecast that $\mathrm{Wi}-\mathrm{Fi}$ and LTE will be among the dominant technologies used by RATs for access purposes over the next few years. Thus, this paper focuses on the coordinated coexistence between these two technologies. LTE is designed to operate solely in a spectrum, which when operating in unlicensed spectrum, is termed LTE-U. It is suggested in 3GPP, that LTE-U will be used as a supplemental downlink, whereas the uplink will use licensed spectrum. This makes the deployment even more challenging as the UE's do not transmit in unlicensed spectrum yet experience interference from $\mathrm{Wi}-\mathrm{Fi}$ transmissions. To alleviate these problems, we extend the interference characterization of co-channel deployment of Wi-Fi and LTE using simplistic but accurate analytical model [9]. Then, we validate this model through experimental analysis of co-channel deployment in the $2.4 \mathrm{GHz}$ band, using the ORBIT testbed and LTE on USRP platforms available at WINLAB.

To support the co-existence of a multi-RAT network, we propose a dynamic spectrum coordination framework, which is enabled by a Software Defined Network (SDN) architecture. $\mathrm{SDN}$ is technology-agnostic, can accommodate different radio standards and does not require change to existing standards or protocols. In contrast to existing technology-centric solutions, this is a desirable feature, especially in the rapid development of upcoming technologies and spectrum bands [10], [11]. Furthermore, the proposed framework takes advantage of the ubiquitous Internet connectivity available at wireless devices and provides the pseudo-global network with the ability to consider policy requirements in conjunction with improved visibility of each of the technologies, spectrum bands, clients and/or operators. Thus, it offers significant benefits for spectrum allocation over centralized spectrum servers [12] or radio based control channels [13].

While the inter-network cooperation enabled by SDN can be used for optimizing several spectrum usage parameters such as power control, channel selection, rate allocation, and duty cycle, in this paper, we focus on power control at both LTE and $\mathrm{Wi}-\mathrm{Fi}$, which maximizes aggregate throughput at all clients across both Wi-Fi and LTE networks along with consideration of throughput requirement at each client [14], [15]. We also propose to apply validated interference characterization of Wi-Fi-LTE coexistence in the optimization framework, which captures the specific requirements of each of the technologies. In general, we adopt the geometric programming framework developed in [16] for the LTE-only network and enhance it to accommodate Wi-Fi network.

The major contributions of this work are as follows:

- We introduce an analytical model to characterize the interference between Wi-Fi and LTE networks, when they coexist and share the medium in time, frequency and space. We have also validated the model by performing experimental analysis using USRP based LTE nodes and commercial off-the-shelf (COTS) IEEE $802.11 \mathrm{~g}$ devices in the ORBIT testbed.

- We propose a coordination framework to facilitate dynamic spectrum management among multi-operator and multi-technology networks over a large geographical area.

- We propose a logically centralized cooperative optimization framework that involves dynamic coordination between Wi-Fi and LTE networks by exploiting power control and time division channel access diversity.

- We evaluate the proposed optimization framework for improved coexistence between $\mathrm{Wi}-\mathrm{Fi}$ and LTE networks.

The rest of the paper is organized as follows. In $\$$ II we discuss previous work on this topic and distinguish our work from existing literature. In $\$$ III. we propose an analytical model to characterize the interference between $\mathrm{Wi}-\mathrm{Fi}$ and LTE networks followed by partial experimental validation of the model. In $[\mathrm{IV}$, we propose an SDN-based inter-network coordination architecture, which can be used for transferring control messages between the different entities in the network. We use two approaches - power control and channel access time sharing methods to jointly optimize the spectrum sharing among Wi-Fi and LTE networks, which is described in \$VI followed by their evaluation in $\$ \mathrm{VII}$. We conclude in $\$ \mathrm{VIII}$.

\section{BACKGROUND ON WI-FI/LTE CO-EXISTENCE}

Coordination between multi-RAT networks with LTE and $\mathrm{Wi}-\mathrm{Fi}$ is challenging due to the difference in the medium access control layer of the two technologies.

Wi-Fi is based on the distributed coordination function (DCF) where each transmitter senses the channel energy for transmission opportunities and collision avoidance. In particular, clear channel assessment (CCA) in Wi-Fi involves two functions to detect any on-going transmissions [17], [18] -

1) Carrier sense: Defines the ability of the Wi-Fi node to detect and decode other nodes' preambles, which most likely announces an incoming transmission. In such cases, Wi-Fi nodes are said to be in the CSMA range of each other other. For the basic DCF with no RTS/CTS, the Wi-Fi throughput can be accurately characterized using 
the Markov chain analysis given in Bianchi's model [19], assuming a saturated traffic condition (at least 1 packet is waiting to be sent) at each node. Wi-Fi channel rates used in the [19] can be modeled as a function of Signalto-Interference-plus-Noise ratio. Our throughput analysis given in the following sections is based on Bianchi's model.

2) Energy detection: Defines the ability of Wi-Fi to detect non-Wi-Fi (in this case, LTE) energy in the operating channel and back off the data transmission. If the inband signal energy crosses a certain threshold, the channel is detected as busy (no Wi-Fi transmission) until the channel energy is below the threshold. Thus, this function becomes the key parameter for characterizing Wi-Fi throughput in the co-channel deployment with LTE.

LTE has both frequency division (FDD) and time division (TDD) multiplexing modes to operate. But to operate in unlicensed spectrum, supplemental downlink and TDD access is preferred. In either of the operations, data packets are scheduled in the successive time frames. LTE is based on orthogonal frequency-division multiple access (OFDMA), where a subset of subcarriers can be assigned to multiple users for a certain symbol time. This gives LTE additional diversity in the time and frequency domain that Wi-Fi lacks, since Wi-Fi bandwidth is assigned to a single user at any time. Further, LTE does not assume that spectrum is shared, and consequently does not employ any sharing features in the channel access mechanisms. Thus, the coexistence performance of both Wi-Fi and LTE is largely unpredictable and may lead to unfair spectrum sharing or the starvation of one of the technologies [20], [21].

In the literature, several studies have discussed spectrum management for multi-RAT heterogeneous networks in shared frequency bands, primarily focusing on IEEE 802.11 and 802.16 networks [11], [13], [22]. Recently, Wi-Fi and LTE coexistence has been studied in the context of TV white space [23], in-device coexistence [24], and LTE-unlicensed (LTE-U) [25]-[27]. Several studies [26]-[28] propose CSMA/sensing based modifications in LTE with features like Listen-beforeTalk, RTS/CTS protocol, and slotted channel access. In other studies, to enable Wi-Fi/LTE coexistence, solutions like blank LTE subframes/LTE muting (feature in LTE Release 10/11) [23], [29], carrier sensing adaptive transmission [26], interference aware power control in LTE [30] have been proposed, which require LTE to transfer its resources to Wi-Fi. These schemes give Wi-Fi transmission opportunities but also lead to performance tradeoffs for LTE. Further, time domain solutions often require time synchronization between Wi-Fi and LTE and increase channel signaling. Some aspects of frequency and LTE bandwidth diversity have been explored in studies [26] and [31], respectively. Frequency diversity is perhaps the least studied problem in Wi-Fi/LTE coexistence, while previous studies also have yet to consider dense Wi-Fi and LTE HetNet deployment scenarios in detail. Notably, in the literature, there are no previous studies experimentally evaluating the coexistence performance of Wi-Fi and LTE.

\section{INTERFERENCE CHARACTERIZATION}

\section{A. Interference Characterization Model}

We propose an analytical model to characterize the interference between Wi-Fi and LTE, while considering the Wi-
Fi sensing mechanism (clear channel assessment (CCA)) and scheduled and persistent packet transmission at LTE. To illustrate, we focus on a co-channel deployment involving a single $\mathrm{W}$-iFi and a single LTE cell, which involves disseminating the interaction of both technologies in detail and establish a building block to study a complex co-channel deployment of multiple Wi-Fis/LTEs.

In a downlink deployment scenario, a single client and a full buffer (saturated traffic condition) is assumed at each AP under no MIMO. Transmit powers are denoted as $P_{i}, i \in$ $\{w, l\}$ where $w$ and $l$ are indices to denote Wi-Fi and LTE links, respectively. We note that the maximum transmission power of an LTE small cell is comparable to that of the Wi$\mathrm{Fi}$, and thus is consistent with regulations of unlicensed bands.

The power received from a transmitter $j$ at a receiver $i$ is given by $P_{j} G_{i j}$ where $G_{i j} \geq 0$ represents a channel gain which is inversely proportional to $d_{i j}^{\gamma}$ where $d_{i j}$ is the distance between $i$ and $j$ and $\gamma$ is the path loss exponent. $G_{i j}$ may also include antenna gain, cable loss, wall loss, and other factors. Signal-to-Interference-plus-Noise (SINR) on the link $i$ given as

$$
S_{i}=\frac{P_{i} G_{i i}}{P_{j} G_{i j}+N_{i}}, \quad i, j \in\{w, l\}, i \neq j
$$

where $N_{i}$ is noise power for receiver $i$. Here, in the case of a single Wi-Fi and LTE, if $i$ represents the Wi-Fi link, then $j$ is the LTE link, and vice versa.

The throughput, $R_{i}, i \in\{w, l\}$, can be represented as a function of $S_{i}$ as

$$
R_{i}=\alpha_{i} B \log _{2}\left(1+\beta_{i} S_{i}\right), \quad i \in\{w, l\},
$$

where $B$ is a channel bandwidth; $\beta_{i}$ is a factor associated with the modulation scheme. For LTE, $\alpha_{l}$ is a bandwidth efficiency due to factors adjacent channel leakage ratio and practical filter, cyclic prefix, pilot assisted channel estimation, signaling overhead, etc. For Wi-Fi, $\alpha_{w}$ is the bandwidth efficiency of CSMA/CA, which comes from the Markov chain analysis of CSMA/CA [19] with

$$
\eta_{E}=\frac{T_{E}}{\mathrm{E}[S]}, \eta_{S}=\frac{T_{S}}{\mathrm{E}[S]}, \eta_{C}=\frac{T_{C}}{\mathrm{E}[S]},
$$

where $\mathrm{E}[S]$ is the expected time per Wi-Fi packet transmission; $T_{E}, T_{S}, T_{C}$ are the average times per $\mathrm{E}[S]$ that the channel is empty due to random backoff, or busy due to the successful transmission or packet collision (in case of multiple Wi-Fis in the CSMA range), respectively. $\alpha_{w}$ is mainly associated with $\eta_{S}$.

In our analysis, $\left\{\alpha_{i}, \beta_{i}\right\}$ is approximated so that - (1) for LTE, $R_{l}$ matches with throughput achieved under variable channel quality index (CQI), and (2) for Wi-Fi, $R_{w}$ matches throughput achieved under Biachi's CSMA/CA model.

1) Characterization of Wi-Fi Throughput: Assuming $\lambda_{c}$ is CCA threshold to detect channel as busy or not, if channel energy at the Wi-Fi node is higher than $\lambda_{c}$, Wi-Fi would hold back the data transmission, otherwise it transmit at a data rate based on the SINR of the link. Wi-Fi throughput with and without LTE is given as

\footnotetext{
${ }^{1}$ Throughput the paper, LTE home-eNB (HeNB) is also referred as access point (AP) for the purpose of convenience
} 


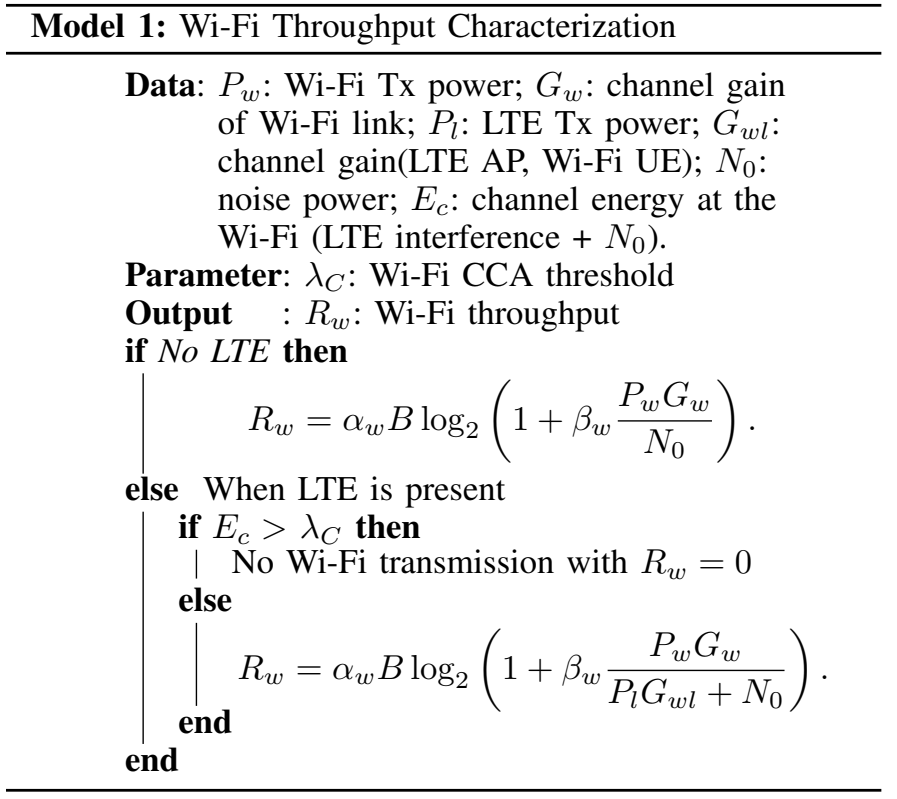

2) Characterization of LTE Throughput: Due to CSMA/CA, Wi-Fi is active for an average $\eta_{S}$ fraction of time (Eq. (3)). Assuming that LTE can instantaneously update its transmission rate based on the Wi-Fi interference, its throughput can be modeled as follows-

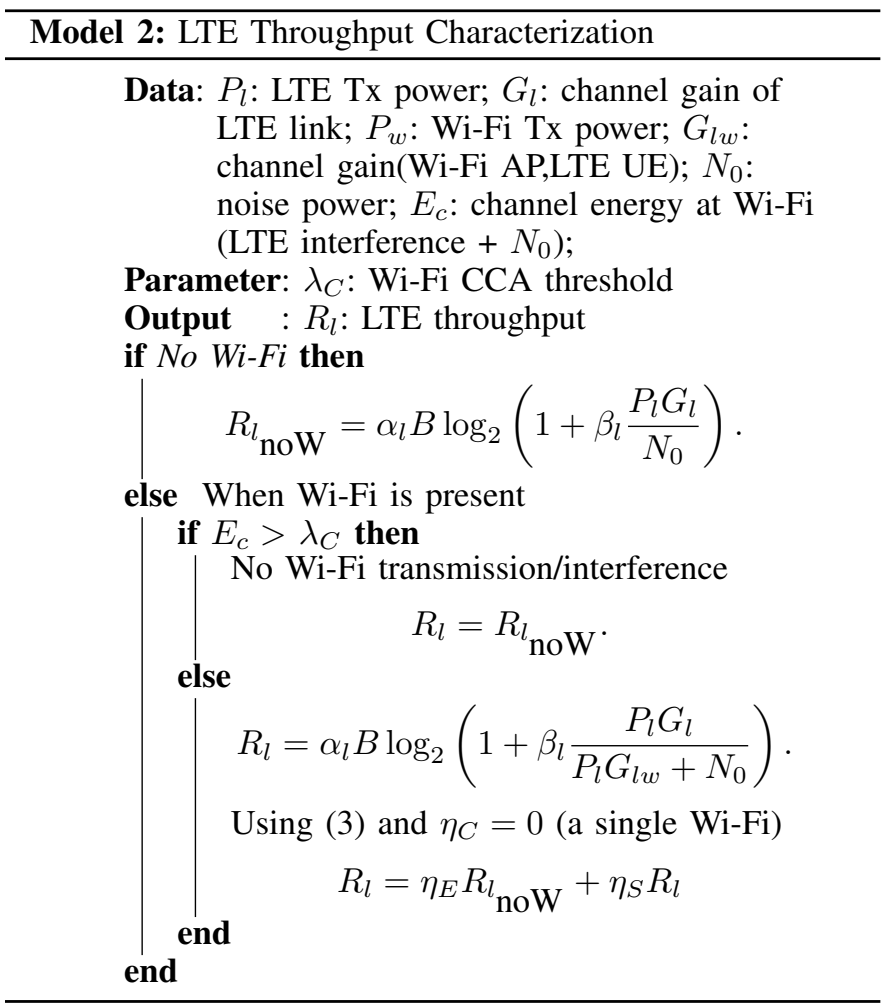

\section{B. Experimental Validation}

In this section, we experimentally validate proposed interference characterization models using experiments involving the ORBIT testbed and USRP radio platforms available at WINLAB [32], [33]. An 802.11g Wi-Fi link is set up

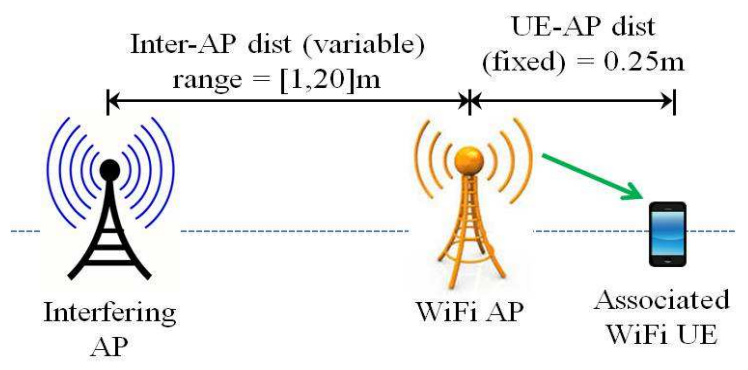

Fig. 3. Experimental scenario to evaluate the throughput performance of Wi-Fi $w_{1}$ in the presence of interference (LTE/other Wi-Fi/white noise) when both $w_{1}$ and interference operated on the same channel in $2.4 \mathrm{GHz}$

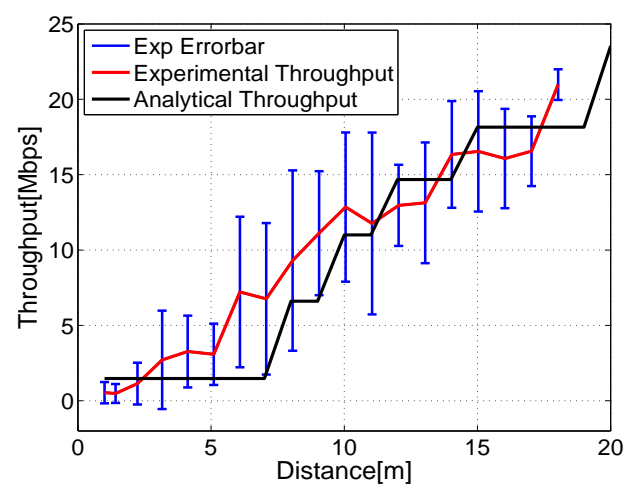

Fig. 4. Comparative results analytical model and experiments to show the effect of LTE on the throughput of Wi-Fi 802.11g when distance between LTE eNB and Wi-Fi link is varied.

using Atheros AR928X wireless network adapters [34] and an AP implementation with hostapd [35]. For LTE, we use OpenAirInterface, an open-source software implementation, which is fully compliant with 3GPP LTE standard (release 8.6) and set in transmission mode 1 (SISO) [36]. Currently, OpenAirInterface is in the development mode for USRP based platforms with limited working LTE operation parameters.

In our experiment, depicted as the scenario shown in figure 3, we study the effect of interference on the Wi-Fi link $w_{1}$. For link $w_{1}$, the distance between the AP and client is fixed at $0.25 \mathrm{~m}$ (very close so that the maximum throughput is guaranteed when interference is present. Experimentally, we observe maximum throughput as $22.2 \mathrm{Mbps}$ ). The distance between the interfering $\mathrm{AP}$ and $\mathrm{Wi}-\mathrm{Fi} \mathrm{AP}$ is varied in the range of 1 to $20 \mathrm{~m}$. The throughput of $w_{1}$ is evaluated under three sources of interference - LTE and Wi-Fi, when both $w_{1}$ and the interference AP is operated on the same channel in the $2.4 \mathrm{GHz}$ spectrum band. These experiments are carried in the $20 \mathrm{~m}$-by$20 \mathrm{~m}$ ORBIT room in WINLAB, which has an indoor Lineof-Sight (LoS) environment. For each source of interference, Wi-Fi throughput is averaged over 15 sets of experiments with variable source locations and trajectories between interference and $w_{1}$.

In the first experiment, we perform a comparison study to evaluate the effect of LTE interference on $w_{1}$, observed by experiments and computed by interference characterization model. In this case, LTE signal is lightly loaded on $5 \mathrm{MHz}$ of bandwidth mainly consist of control signals. Thus, the impact 


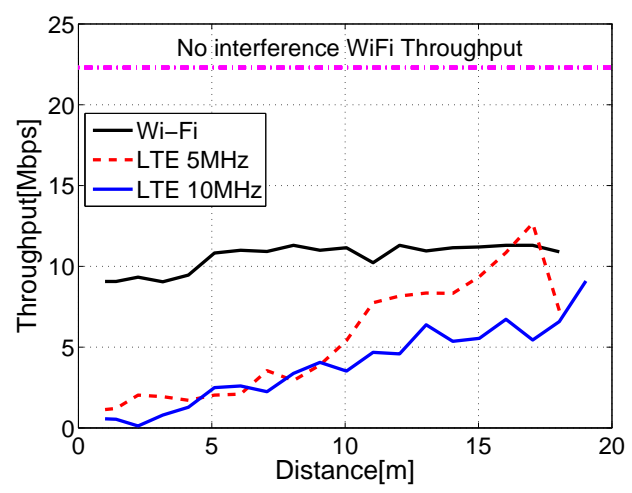

Fig. 5. Comparative results analytical model and experiments to show the effect of LTE on the throughput of Wi-Fi 802.11g when distance between LTE HeNB (AP) and Wi-Fi link is varied.

TABLE I. NETWORK PARAMETERS OF WI-FI/LTE DEPLOYMENT

\begin{tabular}{|l|l|l|l|}
\hline Parameter & Value & Parameter & Value \\
\hline Scenario & Downlink & Tx power & $20 \mathrm{dBm}$ \\
\hline Spectrum band & $2.4 \mathrm{GHz}$ & Channel bandwidth & $20 \mathrm{MHz}$ \\
\hline Traffic model & Full buffer via saturated UDP flows \\
\hline AP antenna height & $10 \mathrm{~m}$ & User antenna height & $1 \mathrm{~m}$ \\
\hline Path loss model & $36.7 \log _{10}(\mathrm{~d}[\mathrm{~m}])+22.7+26 \log _{10}(\mathrm{frq}[\mathrm{GHz}])$ \\
\hline Noise Floor & $-101 \mathrm{dBm},(-174 \mathrm{cBm}$ thermal noise/Hz) \\
\hline Channel & No shadow/Rayleigh fading \\
\hline Wi-Fi & $802.11 \mathrm{n}$ : SISO \\
\hline LTE & FDD, Tx mode-1 (SISO) \\
\hline
\end{tabular}

of such LTE signal over the Wi-Fi band is equivalent to the low power LTE transmission. Thus, we incorporate these LTE parameters in our analytical model. As shown in figure 4 , we observe that both experimental and analytical values match the trend very closely, though with some discrepancies. These discrepancies are mainly due to the fixed indoor experiment environment and lack of a large number of experimental data sets. Additionally, we note that even with the LTE control signal (without any scheduled LTE data transmission), performance of Wi-Fi gets impacted drastically.

In the next set of experiments, we study the throughput of a single Wi-Fi link in the presence of different sources of interference - (1) Wi-Fi, (2) LTE operating at $5 \mathrm{MHz}$, and (3) LTE operating at $10 \mathrm{MHz}$, evaluating each case individually. For this part, full-band occupied LTE is considered with the maximum power transmission of $100 \mathrm{~mW}$. As shown in figure 5, when the Wi-Fi link operates in the presence of other Wi-Fi links, they share channel according to the CSMA/CA protocol and throughput is reduced approximately by half. In the both the cases of LTE operating at 5 and $10 \mathrm{MHz}$, due to lack of coordination, Wi-Fi throughput gets impacted by maximum upto $90 \%$ compared to no interference Wi-Fi throughput and 20-80\% compared to Wi-Fi thorughput in the presence of other Wi-Fi link. These results indicate significant inter-system interference in the baseline case without any coordination between systems.

\section{Motivational Example}

We extend our interference model to complex scenarios involving co-channel deployment of a single link Wi-Fi and LTE for the detailed performance evaluation. As shown in figure 6 $\mathrm{UE}_{i}$, associated $\mathrm{AP}_{i}$ and interfering $\mathrm{AP}_{j}, i, j \in\{w, l\}, i \neq j$,

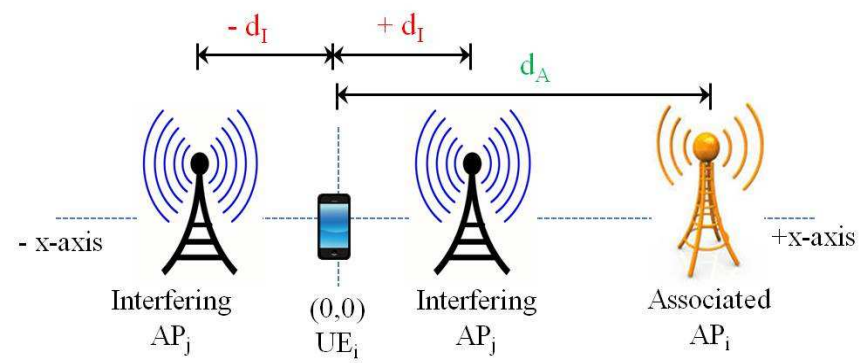

Fig. 6. Experimental scenario to evaluate the throughput performance of Wi-Fi $w_{1}$ in the presence of interference (LTE/other Wi-Fi/white noise) when both $w_{1}$ and interference operated on the same channel in $2.4 \mathrm{GHz}$

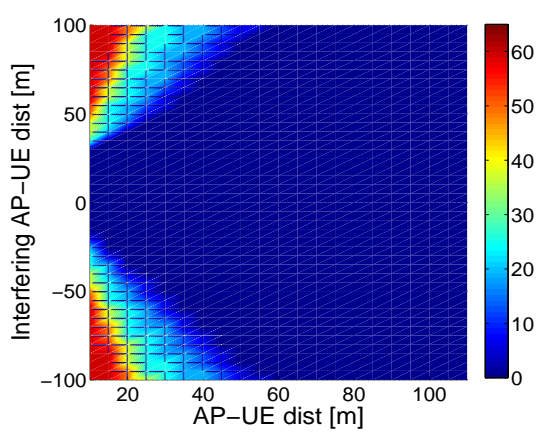

(a) A heat map of Wi-Fi throughput (Mbps)

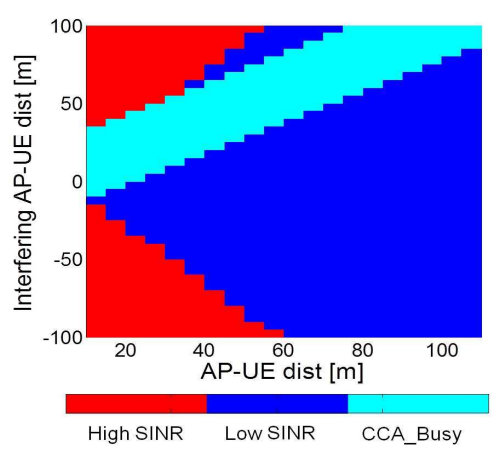

(b) Wi-Fi performance sections- High SINR: non-zero throughput, Low SINR: SINR below minimum SINR requirement, CCA_busy: shutting off of Wi-Fi due to channel is sensed as busy

Fig. 7. Wi-Fi performance as a function of distance(Wi-Fi AP, associated Wi-Fi UE) $d_{A}$ and distance(Interfering LTE AP, Wi-Fi UE) $d_{I}$

are deployed in a horizontal alignment. The distance, $d_{A}$, between $\mathrm{UE}_{i}$ and $\mathrm{AP}_{i}$ is varied between 0 and $100 \mathrm{~m}$. At each value of $d_{A}$, the distance between $\mathrm{UE}_{i}$ and $\mathrm{AP}_{j}$ is varied in the range of -100 to $100 \mathrm{~m}$. Assuming $\mathrm{UE}_{i}$ is located at the origin $(0,0)$, if $\mathrm{AP}_{j}$ is located on the negative $\mathrm{X}$-axis then the distance is denoted as $-d_{I}$, otherwise as $+d_{I}$, where $d_{I}$ is an Euclidean norm $\left\|\mathrm{UE}_{i}, \mathrm{AP}_{j}\right\|$. In the shared band operation of Wi-Fi and LTE, due to the CCA sensing mechanism at the Wi-Fi node, the distance between Wi-Fi and LTE APs (under no shadow fading effect in this study) decides the transmission or shutting off of Wi-Fi. Thus, the above distance convention is adopted to embed the effect of distance between $\mathrm{AP}_{i}$ and $\mathrm{AP}_{j}$. Simulation parameters for this set of simulations are given in Table I. 


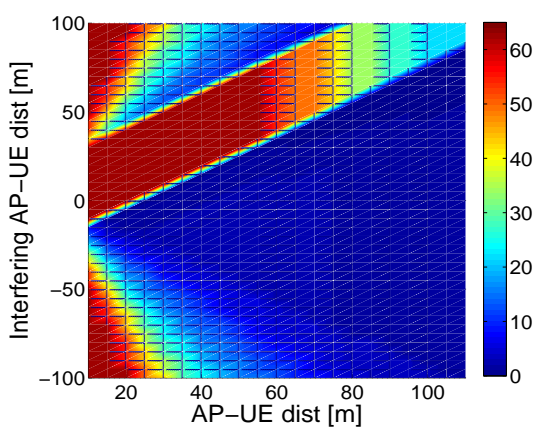

(a) A heat map of LTE throughput (Mbps)

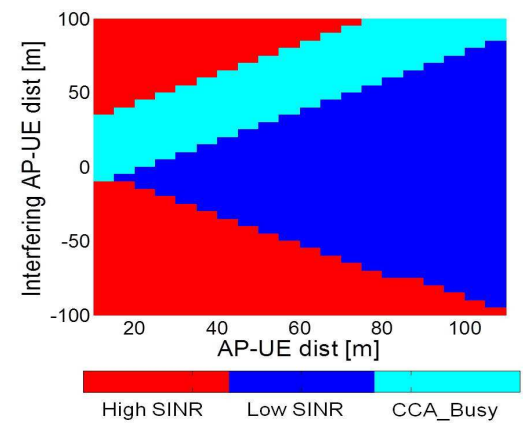

(b) LTE performance sections- High SINR: non-zero throughput, Low SINR: SINR below minimum SINR requirement, CCA_busy: shutting off of Wi-Fi due to channel is sensed as busy

Fig. 8. LTE performance as a function of distance(LTE AP, associated LTE UE) $d_{A}$ and distance(Interfering Wi-Fi AP, LTE UE) $d_{I}$

Figure 7 shows the Wi-Fi performance in the presence of LTE interference. As shown in figure 7(a), the Wi-Fi throughput is drastically deteriorated in the co-channel LTE operation, leading to zero throughput for $80 \%$ of the cases and an average $91 \%$ of throughput degradation compared to standalone operation of Wi-Fi. Such degradation is explained by figure 7(b) Region CCA_busy shows the shutting off of the Wi-Fi AP due to the CCA mechanism, where high energy is sensed in the Wi-Fi band. This region corresponds to cases when Wi-Fi and LTE APs are within $\sim 20 \mathrm{~m}$ of each other. In the low SINR region, the Wi-Fi link does not satisfy the minimum SINR requirement for data transmission, thus the Wi-Fi throughput is zero. High SINR depicts the data transmission region that satisfies SINR and CCA requirements and throughput is varied based on variable data rate/SINR.

On the other hand, figure 8 depicts the LTE throughput in the presence of Wi-Fi interference. LTE throughput is observed to be zero in the low SINR regions, which is $45 \%$ of the overall area and the average throughput degradation is $65 \%$ compared to the standalone LTE operation. Under identical network parameters, overall performance degradation for LTE is much lower compared to that of $\mathrm{Wi}-\mathrm{Fi}$ in the previous example. The reasoning for such a behavior discrepancy is explained with respect to figure $8(\mathrm{~b})$ and the Wi-Fi CCA mechanism. In the CCA_busy region, Wi-Fi operation is shut off and LTE operates as if no Wi-Fi is present. In both LTE and the previous WiFi examples, low SINR represents the hidden node problem where two APs do not detect each other's presence and data transmission at an UE suffers greatly.

\section{System ARChitecture}

In this section, we describe an architecture for coordinating between multiple heterogeneous networks to improve spectrum utilization and facilitate co-existence [10]. Figure 9 shows the proposed system, which is built on the principles of a Software Defined Networking (SDN) architecture to support logicallycentralized dynamic spectrum management involving multiple autonomous networks. The basic design goal of this architecture is to support the seamless communication and information dissemination required for coordination of heterogeneous networks. The system consists of two-tiered controllers: the Global Controller (GC) and Regional Controllers (RC), which are mainly responsible for the control plane of the architecture. The GC, owned by any neutral/authorized organization, is the main decision making entity, which acquires and processes network state information and controls the flow of information between RCs and databases based on authentication and other regulatory policies. Decisions at the GC are based on different network modules, such as radio coverage maps, coordination algorithms, policy and network evaluation matrices. The RCs are limited to network management of specific geographic regions and the GC ensures that RCs have acquired local visibility needed for radio resource allocation at wireless devices. A Local Agent (LA) is a local controller, co-located with an access point or base-station. It receives frequent spectrum usage updates from wireless clients, such as device location, frequency band, duty cycle, power level, and data rate. The signaling between RC and LAs are event-driven, which occurs in scenarios like the non-fulfillment of qualityof-service (QoS) requirements at wireless devices, request-forupdate from an $\mathrm{RC}$ and radio access parameter updates from an $\mathrm{RC}$. The key feature of this architecture is that the frequency of signaling between the different network entities is less in higher tiers compared to lower tiers. RCs only control the regional messages and only wide-area network level signalling protocols are handled at the higher level, GC. Furthermore, this architecture allows adaptive coordination algorithms based on the geographic area and change in wireless device density and traffic patterns. We use this architecture to exchange control messages required for the optimization model, as described in छI

\section{System Model}

As seen in the previous section, when two (or more) APs of different Wi-Fi and LTE networks are deployed in the same spectrum band, APs can cause severe interference to one another. In order to alleviate inter-network interference, we propose joint coordination based on (1) power, and (2) time division channel access optimization. We assume that both LTE and Wi-Fi share a single spectrum channel and operate on the same amount of bandwidth. We also note that clients associated to one AP cannot join other Wi-Fi or LTE APs. This is a typical scenario when multiple autonomous operators deploy APs in the shared band. With the help of the proposed SDN architecture, power level and time division channel access parameters are forwarded to each network based on the throughput requirement at each UE. To the best of our knowledge, such an optimization framework has not yet 


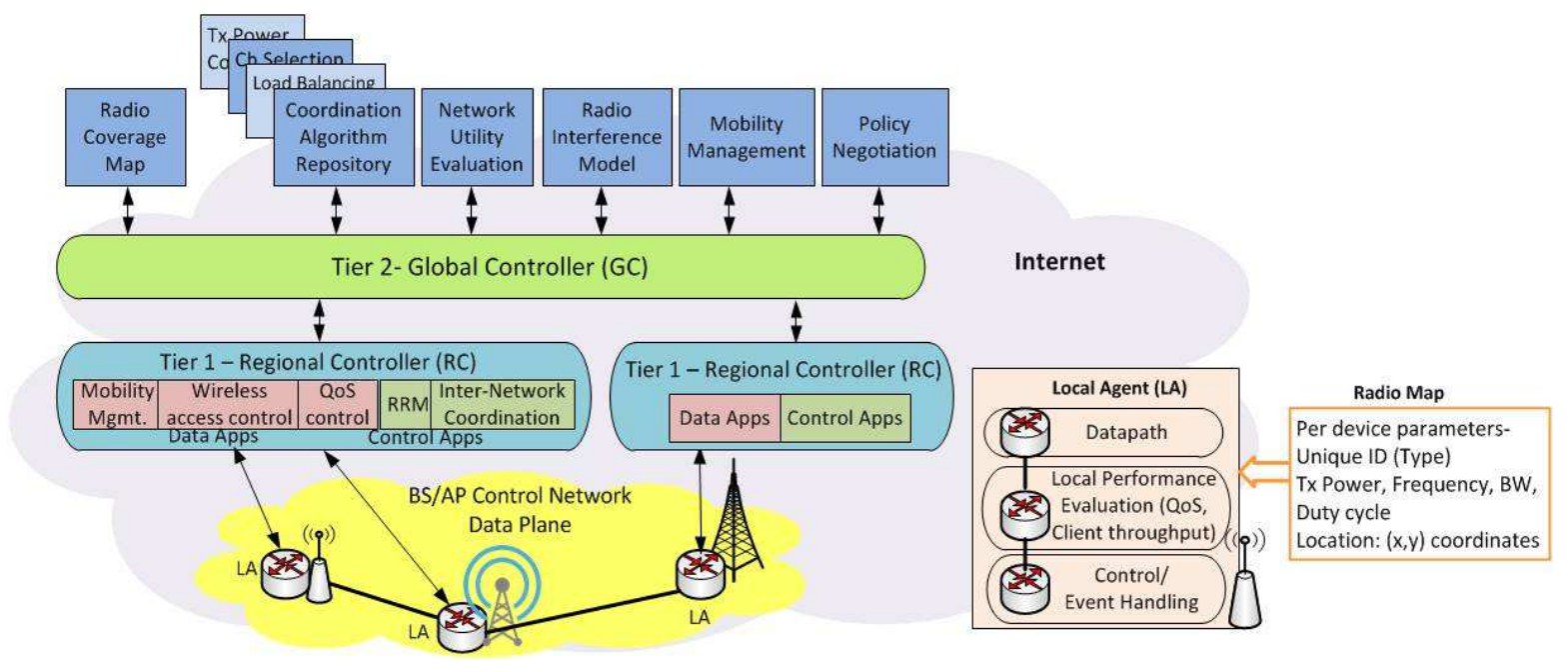

Fig. 9. SDN based achitecture for inter-network cooperation on radio resource management

TABLE II. DEFINITION OF NOTATIONS

\begin{tabular}{|l|l|}
\hline Notation & Definition \\
\hline$w, l$ & indices for Wi-Fi and LTE network, respectively \\
\hline $\mathcal{W}$ & the set of Wi-Fi links \\
\hline $\mathcal{L}$ & the set of LTE links \\
\hline$P_{i}$ & Transmission power of $i$-th AP, where $i \in\{\mathcal{W}, \mathcal{L}\}$ \\
\hline$G_{i j}$ & Channel gain between nodes $i$ and $j$ \\
\hline$R_{i}$ & Throughput at $i$-th link, where $i \in\{\mathcal{W}, \mathcal{L}\}$ \\
\hline$S_{i}$ & SINR at $i$-th link, where $i \in\{\mathcal{W}, \mathcal{L}\}$ \\
\hline$B$ & Channel Bandwidth \\
\hline$N_{0}$ & Noise level \\
\hline$\alpha_{i}, \beta_{i}$ & Efficiency parameters of system $i \in\{\mathcal{W}, \mathcal{L}\}$ \\
\hline$M_{i}^{a}$ & Set of Wi-Fi APs in the CSMA range of AP $i \in\{\mathcal{W}\}$ \\
\hline$M_{i}^{b}$ & Set of Wi-Fi APs in the interference range of AP $i \in\{\mathcal{W}\}$ \\
\hline$\zeta$ & Hidden node interference parameter \\
\hline$\eta$ & $\begin{array}{l}\text { Fraction of channel access time for network } i, i \in\{w, l\} \text { when } \\
j, j \in\{w, l\}, j \neq i, \text { access channel for } 1-\eta \text { fraction of time }\end{array}$ \\
\hline
\end{tabular}

received much attention for the coordination between $\mathrm{Wi}-\mathrm{Fi}$ and LTE networks.

We consider a system with $N$ Wi-Fi and $M$ LTE networks. $\mathcal{W}$ and $\mathcal{L}$ denote the sets of Wi-Fi and LTE links, respectively. We maintain all assumptions, definitions and notations as described in Section III-A For notational simplicity, we redefine $R_{i}=\alpha_{i} B \log _{2}\left(1+\beta_{i} S_{i}\right), i \in\{\mathcal{W}, \mathcal{L}\}$ as $R_{i}=$ $\alpha_{i} \log _{2}\left(1+\beta_{i} S_{i}\right)$, where constant parameter $B$ is absorbed with $\alpha_{i}$. Additional notation are summarized in Table III

In order to account for the co-channel deployment of multiple Wi-Fi networks, we assume that time is shared equally when multiple Wi-Fi APs are within CSMA range due to the Wi-Fi MAC layer. We denote the set of $\mathrm{Wi}-\mathrm{Fi}$ APs within the CSMA range of $\mathrm{AP}_{i}, i \in\{\mathcal{W}\}$ as $M_{i}^{a}$ and those outside of carrier sense but within interference range as $M_{i}^{b}$. When $\mathrm{AP}_{i}$ shares the channel with $\left|M_{i}^{a}\right|$ other APs, its share of the channel access time get reduced to approximately $1 /\left(1+\left|M_{i}^{a}\right|\right)$. Furthermore, $M_{i}^{b}$ signifies a set of potential hidden nodes for $\mathrm{AP}_{i}, \forall i$. To capture the effect of hidden node interference from APs in the interference range, parameter $\zeta$ is introduced which lowers the channel access time and thus, the throughput. Average reduction in channel access time at $\mathrm{AP}_{i}$ is $1 /\left(1+\zeta\left|M_{i}^{b}\right|\right)$ where $\zeta$ falls in the range $[0.2,0.6][37]$. Therefore, the effective Wi-Fi throughput can be written as

$$
\begin{aligned}
R_{i} & =a_{i} b_{i} \alpha_{w} \log _{2}\left(1+\beta_{w} S_{i}\right), \quad i \in \mathcal{W}, \\
\text { with } a_{i} & =\frac{1}{1+\left|M_{i}^{a}\right|} \text { and } b_{i}=\frac{1}{1+\zeta\left|M_{i}^{b}\right|} .
\end{aligned}
$$

SINR of Wi-Fi link, $i, i \in \mathcal{W}$, in the presence of LTE and no LTE is described as

$$
S_{i}= \begin{cases}\frac{P_{i} G_{i i}}{N_{0}}, & \text { if no LTE; } \\ \frac{P_{i} G_{i i}}{\sum_{j \in \mathcal{L}} P_{j} G_{i j}+N_{0}}, & \text { if LTE }\end{cases}
$$

where the term $\sum_{j \in L} P_{j} G_{i j}$ is the interference from all LTE networks at a Wi-Fi link $i$.

The throughput definition of the LTE link $i, i \in \mathcal{L}$ remains the same as in Section III-A.

$$
R_{i}=\alpha_{l} \log _{2}\left(1+\beta_{l} S_{i}\right), \quad i \in \mathcal{L} .
$$

The SINR of the LTE link, $i, \forall i$, in the presence of Wi-Fi and no $\mathrm{Wi}-\mathrm{Fi}$ is described as

$$
S_{i}=\left\{\begin{array}{l}
\frac{P_{i} G_{i i}}{\sum_{j \in \mathcal{L}, j \neq i} P_{j} G_{i j}+N_{0}}, \text { if no Wi-Fi; } \\
\frac{P_{i} G_{i i}}{\sum_{j \in \mathcal{L}, j \neq i} P_{j} G_{i j}+\sum_{k \in \mathcal{W}} a_{k} P_{k} G_{i k}+N_{0}}, \text { if Wi-Fi, }
\end{array}\right.
$$

where terms $\sum_{j \in \mathcal{L}, j \neq i} P_{j} G_{i j}$ and $\sum_{k \in \mathcal{W}} a_{k} P_{k} G_{i k}$ signifies the interference contribution from other LTE links and Wi-Fi links, (assuming all links in $\mathcal{W}$ are active). For the $k$-th $\mathrm{Wi}-\mathrm{Fi}$ link, $\forall k$, the interference is reduced by a factor $a_{k}$ to capture the fact that the $k$-th $\mathrm{Wi}-\mathrm{Fi}$ is active approximately for only $a_{k}$ fraction of time due to the CSMA/CA protocol at Wi-Fi.

For a given model, inter-network coordination is employed to assure a minimum throughput requirement, thus the guaranteed availability of the requested service at each UE. For this purpose, we have implemented our optimization in two stages as described in following subsections. 


\section{COORDINATION VIA JOINT OPTIMIZATION}

\section{A. Joint Power Control Optimization}

Here, the objective is to optimize the set of transmission power $P_{i}, i \in\{\mathcal{W}, \mathcal{L}\}$ at Wi-Fi and LTE APs, which maximizes the aggregated Wi-Fi+LTE throughput. Conventionally, LTE supports the power control in the cellular network. By default, commercially available Wi-Fi APs/routers are set to maximum level [38]. But adaptive power selection capability is incorporated in available $802.11 \mathrm{a} / \mathrm{g} / \mathrm{n} \mathrm{Wi}$-Fi drivers, even though it is not invoked very often. Under the SDN architecture, transmission power level can be made programmable to control the influence of interference from any AP at neighboring radio devices based on the spectrum parameters [39].

For the maximization of aggregated throughput, we propose a geometric programming (GP) based power control [16]. For the problem formulation, throughput, given by Eq. 2, can approximated as

$$
R_{i}=\alpha_{i} \log _{2}\left(\beta_{i} S_{i}\right), \quad i \in\{\mathcal{W}, \mathcal{L}\} .
$$

This equation is valid when $\beta_{i} S_{i}$ is much higher than 1 . In our case, this approximation is reasonable considering minimum SINR requirements for data transmission at both Wi-Fi and LTE. The aggregate throughput of the WiFi+LTE network is

$$
\begin{aligned}
\mathbb{R} & =\sum_{i \in \mathcal{W}} a_{i} b_{i} \alpha_{w} \log _{2}\left(\beta_{w} S_{i}\right)+\sum_{j \in \mathcal{L}} \alpha_{l} \log _{2}\left(\beta_{l} S_{j}\right) \\
& =\log _{2}\left[\left(\prod_{i \in \mathcal{W}}\left(\beta_{w} S_{i}\right)^{a_{i} b_{i} \alpha_{w}}\right)\left(\prod_{j \in \mathcal{L}}\left(\beta_{l} S_{i}\right)^{\alpha_{l}}\right)\right] .
\end{aligned}
$$

In the coordinated framework, it is assumed that $\mathrm{WiFi}$ parameters $a_{i}$ and $b_{i}$ are updated periodically. Thus, these are considered as constant parameters in the formulation. Also, $\alpha_{i}, \beta_{i}, i \in\{w, l\}$ are constant in the network. Therefore, aggregate throughput maximization is equivalent to maximization of a product of SINR at both WiFi and LTE links. Power control optimization formulation is given by:

$$
\begin{array}{ll}
\operatorname{maximize} & \left(\prod_{i \in \mathcal{W}}\left(\beta_{w} S_{i}\right)^{a_{i} b_{i} \alpha_{w}}\right)\left(\prod_{j \in \mathcal{L}}\left(\beta_{l} S_{i}\right)^{\alpha_{l}}\right) \\
\text { subject to } \quad & R_{i} \geq R_{i, \min }, \quad i \in \mathcal{W}, \\
& R_{i} \geq R_{i, \min }, \quad i \in \mathcal{L}, \\
& \sum_{k \in M_{i}^{b}} P_{k} G_{i k}+\sum_{j \in \mathcal{L}} P_{j} G_{i j}+N_{0}<\lambda_{c}, i \in W, \\
& 0<P_{i} \leq P_{\max }, \quad i \in \mathcal{W}, \\
& 0<P_{i} \leq P_{\max }, \quad i \in \mathcal{L} .
\end{array}
$$

Here, the first and second constraints are equivalent to $S_{i} \geq$ $S_{i, \min }, \forall i$ which ensures that SINR at each link achieves a minimum SINR requirement, thus leading to non-zero throughput at the UE. The third constraint assures that channel energy at a WiFi (LTE interference + interference from WiFis in the interference zone + noise power) is below the clear channel assessment threshold $\lambda_{c}$, thus $\mathrm{WiFi}$ is not shut off. The fourth and fifth constraints follow the transmission power limits at each link. Unlike past power control optimization formulations for cellular networks, WiFi-LTE coexistence requires to meet the SINR requirement at a WiFi UE and, additionally, CCA threshold at a WiFi AP.

For multiple Wi-Fi and LTE links, to ensure the feasibility of the problem where all constrains are not satisfied, notably for WiFi links, we relax the minimum data requirement constraint for LTE links. In our case, we reduce the minimum data requirement to zero. This is equivalent to shutting off certain LTE links which cause undue interference to neighboring WiFi devices.

\section{B. Joint Time Division Channel Access Optimization}

The relaxation of minimum throughput constraint in the joint power control optimization leads to throughput deprivation at some LTE links. Thus, joint power control is not sufficient when system demands to have non-zero throughput at each UE. In such cases, we propose a time division channel access optimization framework where network of each RAT take turns to access the channel. Assuming network $i, i \in\{w, l\}$ access the channel for $\eta$, eta $\in[0,1]$, fraction of time, network $j, j \in\{w, l\}, j \neq i$, holds back the transmission and thus no interference occurs at $i$ from $j$. For remaining $1-\eta$ fraction of time, $j$ access the channel without any interference from $i$. This proposed approach can be seen as a subset of power assignment problem, where power levels at APs of network $i, i \in\{w, l\}$, is set to zero in their respective time slots. The implementation of the protocol is out of scope of this paper.

In this approach, our objective is to optimize $\eta$, the time division of channel access, such that it maximizes the minimum throughput across both WiFi and LTE networks. We propose the optimization in two steps -

1) Power control optimization across network of same RAT: Based on the GP-formulation, the transmission power of the APs across the same network $i, i \in\{w, l\}$, are optimized such that

$$
\begin{aligned}
& \text { maximize } \sum_{i \in \mathcal{W}} R_{i} \\
& \text { subject to } R_{i} \geq R_{i, \min }, \quad i \in \mathcal{W} \\
& 0 \leq P_{i} \leq P_{\max }, \quad i \in \mathcal{W} \text {, } \\
& \sum_{k \in M_{i}^{b}} P_{k} G_{i k}+N_{0}<\lambda_{c}, i \in \mathcal{W} \text {. }
\end{aligned}
$$

and

$$
\begin{array}{ll}
\operatorname{maximize} & \sum_{i \in \mathcal{L}} R_{i} \\
\text { subject to } & R_{i} \geq R_{i, \min }, \quad i \in \mathcal{L} \\
& 0 \leq P_{i} \leq P_{\max }, \quad i \in \mathcal{L} .
\end{array}
$$

Here, the objective function is equivalent to maximizing the product of SINRs at the networks $i, i \in\{w, l\}$. The first and second constraints ensure that we meet the minimum SINR and transmission power limits requirements at all links of $i$. In this formulation, SINR at $\mathrm{WiFi}$ and LTE respectively given as

$$
\begin{aligned}
S_{i} & =\frac{P_{i} G_{i i}}{N_{0}}, \quad i \in \mathcal{W}, \\
S_{i} & =\frac{P_{i} G_{i i}}{\sum_{j \in \mathcal{L}, j \neq i} P_{j} G_{i j}+N_{0}}, \quad i \in \mathcal{L} .
\end{aligned}
$$

which are first cases in equations (5) and (6), respectively. 


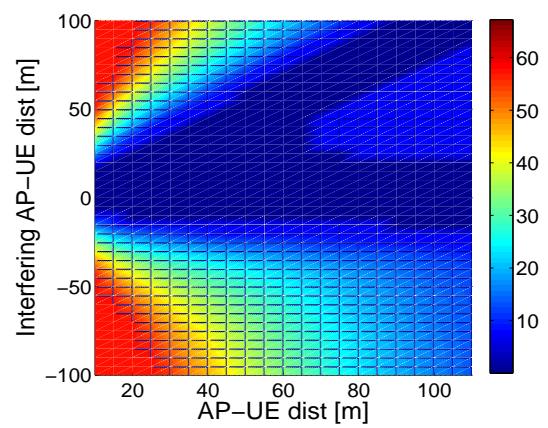

(a) A heat map of WiFi throughput when joint power Coordination (Mbps)

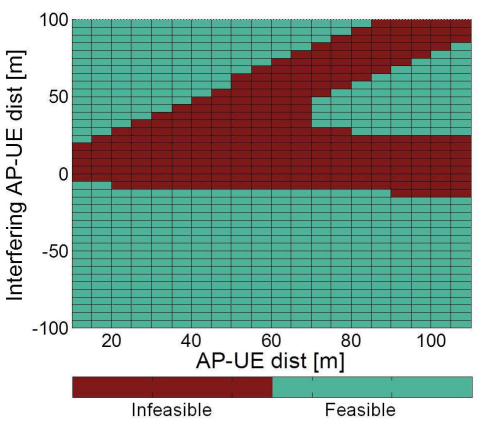

(b) Feasibility region of joint power Coordination

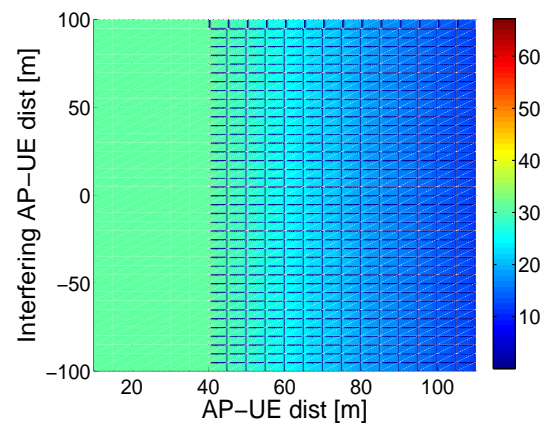

(c) A heat map of WiFi throughput when time division channel access coordination (Mbps)

Fig. 10. WiFi performance under joint WiFi and LTE power control optimization

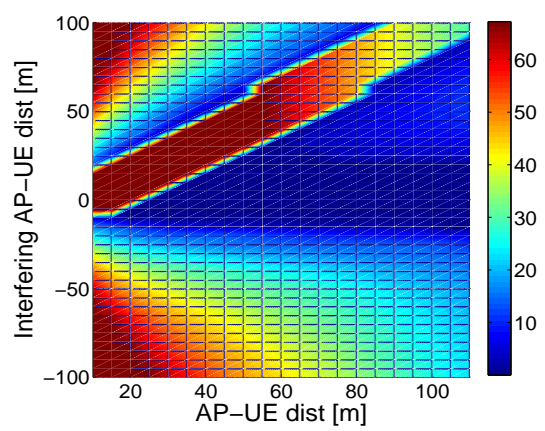

(a) A heat map of LTE throughput when joint power Coordination (Mbps)

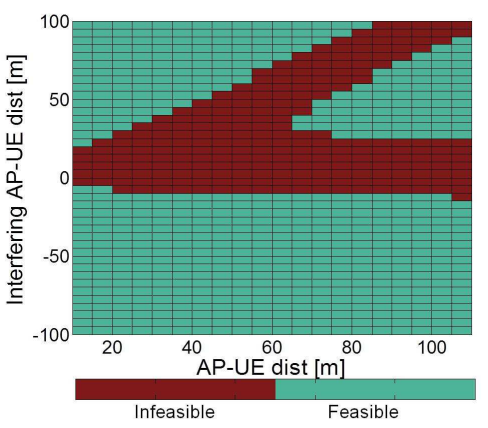

(b) Feasibility region of joint power Coordination

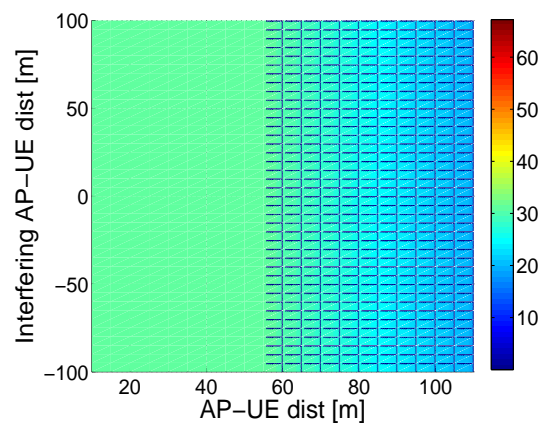

(c) A heat map of LTE throughput when time division channel access coordination (Mbps)

Fig. 11. WiFi performance under joint WiFi and LTE power control optimization

2) Joint time division channel access optimization: This is the joint optimization across both WiFi and LTE networks which is formulated as given below

$$
\begin{array}{ll}
\text { maximize } & \min \left(\eta R_{i \in \mathcal{W}},(1-\eta) R_{j \in \mathcal{L}}\right) \\
\text { subject to } & 0 \leq \eta \leq 1 .
\end{array}
$$

Here, throughput values at all $\mathrm{WiFi}$ and LTE nodes are considered as a constant, which is the output of the previous step. Time division channel access parameter $\eta$ is optimized so that it maximizes the minimum throughput across all UEs.

\section{EVALUATION OF JOINT COORDINATION}

\section{A. Single Link Co-channel Deployment}

We begin with the motivational example of co-channel deployment of one Wi-Fi and one LTE links, as described in $\S$ III-C Figure 10 shows the heatmap of improved throughput of Wi-Fi link, when joint Wi-Fi and LTE coordination is provided in comparison with the throughput with no coordination as shown in figure 7. Similarly, figure 11 shows the heatmap of improved throughput of LTE link, when joint coordination is provided in comparison with the throughput with no coordination, as shown in figure 8

For both the figures 10 and 11 , in their respective scenarios, though joint power control improves the overall throughput for most of topological scenarios (see Figure (a) of 10 and 11), it is not an adequate solution for topological combination marked by infeasible region as given in figure (b) of 10 and 11. The infeasible region signifies the failure to attain the CCA threshold at Wi-Fi AP and link SINR requirement when the UE and interfering AP are very close to each other. When we apply time division channel access optimization for a given scenario, we do not observe any infeasible region, in fact optimization achieves almost equal and fair throughput at both Wi-Fi and LTE link, as shown in figure (c) of 10 and 11. On the downside, this optimization does not consider cases when WiFi and LTE links can operate simultaneously without causing severe interference to each other. In such cases, throughput at both Wi-Fi and LTE get degraded.

Figure 12 summarizes the performance of $\mathrm{Wi}-\mathrm{Fi}$ and LTE links in terms of 10 percentile and mean throughput. We note that the 10 percentile throughput of both Wi-Fi and LTE is increased to $15-20 \mathrm{Mbps}$ for time division coordination compared to $\sim$ zero throughput for no and power coordination. We observe $200 \%$ and $350 \%$ Wi-Fi mean throughput gains due to power and time division channel access, respectively, compared to no coordination. For LTE, throughput gains for both of these coordination is $\sim 25-30 \%$. It appears that time division channel access coordination does not offer any additional advantage to LTE in comparison with power coordination. But it brings the throughput fairness between 


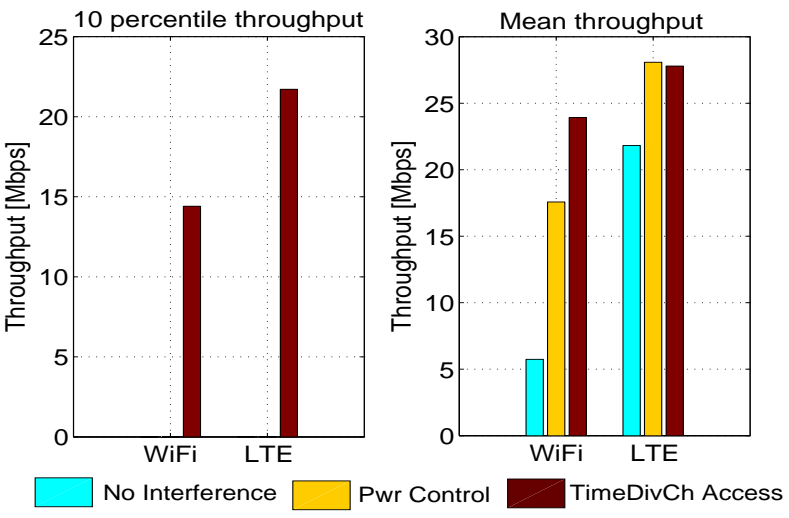

Fig. 12. 10 percentile and mean LTE throughput for a single link WiFi and LTE co-channel deployment

Wi-Fi and LTE which is required for the co-existence in the shared band.

\section{B. Multiple Links Co-channel Deployment}

Multiple overlapping Wi-Fi and LTE links are randomly deployed in 200-by-200 sq. meter area which depicts the typical deployment in residential or urban hotspot. The number of APs of each Wi-Fi and LTE networks are varied between 2 to 10 where number of Wi-Fi and LTE links are assumed to be equal. For the simplicity purpose, we assume that only single client is connected at each AP and their association is predefined. The given formulation can be extended for multiple client scenarios. In the simulations, the carrier sense and interference range for Wi-Fi devices are set to 150 meters and 210 meters, respectively. The hidden node interference parameter is set to 0.25 .

Figure 13(a) and 13(a) show the percentile and mean throughput values of Wi-Fi and LTE links, respectively, for when number of links for each Wi-Fi and LTE networks is set at $N=\{2,5,10\}$. The throughput performance is averaged over 10 different deployment topologies of Wi-Fi and LTE links. From figure 13(a), it is clear that 10 percentile Wi-Fi UEs get throughput starved due to LTE interference with no coordination. This is consistent with results from single link simulations. With coordination, both joint power control and time division channel access, we achieve a large improvement in the 10 percentile throughput. Joint power control improves mean Wi-Fi throughput by $15-20 \%$ for all $N$. On the other hand, time division channel access achieves throughput gain (40-60\%) only at higher values of $N=\{5,10\}$.

Throughput performance of LTE, on the other hand, get deteriorates in the presence of coordination compared to when no coordination is provided. This comes from the fact that, in case of no coordination, LTE causes undue impact at Wi-Fi which makes them to hold off data transmission and LTE experiences no Wi-Fi interference. The joint coordination between Wi-Fi and LTE networks brings the notion of fairness in the system and allocates spectrum resources to suffered Wi-Fi links. In the joint power control optimization, though certain LTE links (maximum 1 link for $N=10$ ) have to be

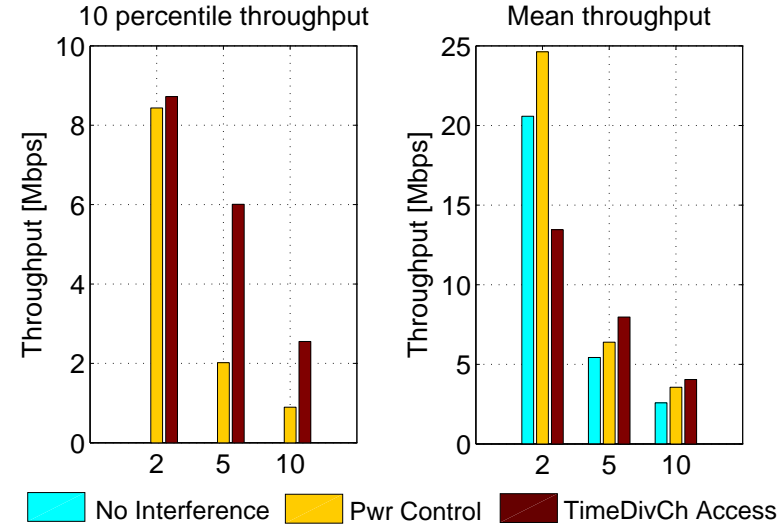

(a) 10 percentile and mean Wi-Fi throughput for $N=\{2,5,10\}$
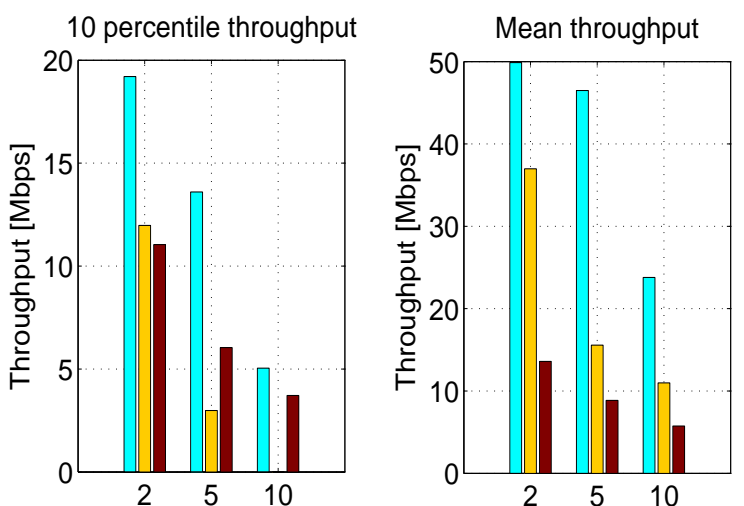

No Interference $\square$ Pwr Control $\square$ TimeDivCh Access

(b) 10 percentile and mean LTE throughput for $N=\{2,5,10\}$

Fig. 13. Multi-link throughput performance under power control and time devision channel access optimization. $N=$ no. of LTE links $=$ no. of $\mathrm{Wi}-\mathrm{Fi}$ links.

dropped from network with zero throughput, the overall mean throughput is greater than 150 to $400 \%$ than Wi-Fi throughput.

We observe that for small numbers of Wi-Fi links, joint time division channel access degrades the performance of both Wi-Fi and LTE. But as number of links grows, coordinated optimization results in allocation of orthogonal resources (e.g. separate channels) gives greater benefit than full sharing of the same spectrum space, as is the case for power control optimization.

\section{CONCLUSION}

This paper investigates inter-system interference in shared spectrum scenarios with both Wi-Fi and LTE in the same band. An analytical model has been developed for evaluation of the performance and the model has been partially verified with experimental data. The results show that significant performance degradation results from uncoordinated operation of Wi-Fi and LTE in the same band. To address this problem, we further presented an architecture for coordination between heterogeneous networks, with a specific focus on LTE-U and $\mathrm{Wi}-\mathrm{Fi}$, to cooperate and coexist in the same area. This framework is used to exchange information between the two 
networks for a logically centralized optimization approach that improves the aggregate throughput of the network. Our results show that, with joint power control and time division multiplexing, the aggregate throughput of each of the networks becomes comparable, thus realizing fair access to the spectrum.

Acknowledgment: Research is supported by NSF EARS program- grant CNS-1247764.

\section{REFERENCES}

[1] “'“Cisco Visual Networking Index: global mobile data traffic forecast update, 2013-2018"," Cisco White Paper, Feb 2014.

[2] Samsung, "Vision and key features for 5th generation (5G) cellular," 2014, http://tinyurl.com/lo5gg53.

[3] Shahram Giri, "Exploring 5G: Performance targets, technologies and timelines," 2014, http://tinyurl.com/mznelg8.

[4] June Kamran Etemad, LTE World Summit, "Improving cell capacity of $5 \mathrm{G}$ systems through opportunistic use of unlicensed and shared spectrum," 2014, http://tinyurl.com/k4bwjw4.

[5] Agilent Technologies Moray Rumney, "Taking 5G from vision to reality," 2014, The 6th Future of Wireless International Conference, http://tinyurl.com/o8hug8j.

[6] RP-140060, "Summary of a workshop on LTE in Unlicensed Spectrum," 3GPP TSG-RAN Meeting 63, 2014.

[7] Intel Corporation Ali Sadri, "mmWave technology evolution from WiGig to 5G small cells," 2013, http://tinyurl.com/lejlgfg.

[8] S.J. Shellhammer, A.K. Sadek, and Wenyi Zhang, "Technical challenges for cognitive radio in the TV white space spectrum," in Information Theory and Applications Workshop, 2009, Feb 2009, pp. 323-333.

[9] S. Sagari, I. Seskar, and D. Raychaudhuri, "Modeling the coexistence of LTE and WiFi heterogeneous networks in dense deployment scenarios," accepted at IEEE ICC workshop 2015 on LTE in Unlicensed Bands: Potentials and Challenges.

[10] D. Raychaudhuri and A. Baid, "NASCOR: Network assisted spectrum coordination service for coexistence between heterogeneous radio systems," IEICE Trans. Commun., vol. 97, no. 2, pp. 251-260, 2014.

[11] G. Nychis, C. Tsourakakis, S. Seshan, and P. Steenkiste, "Centralized, measurement-based, spectrum management for environments with heterogeneous wireless networks," in Dynamic Spectrum Access Networks (DYSPAN), 2014 IEEE International Symposium on, April 2014, pp. 303-314.

[12] O. Ileri, D. Samardzija, and N.B. Mandayam, "Demand responsive pricing and competitive spectrum allocation via a spectrum server," in New Frontiers in Dynamic Spectrum Access Networks, 2005. DySPAN 2005. 2005 First IEEE International Symposium on, Nov 2005, pp. 194-202.

[13] Xiangpeng Jing and Dipankar Raychaudhuri, "Spectrum co-existence of ieee $802.11 \mathrm{~b}$ and $802.16 \mathrm{a}$ networks using reactive and proactive etiquette policies," Mobile Networks and Applications, vol. 11, no. 4, pp. 539-554, 2006.

[14] A. Baid and D. Raychaudhuri, "Understanding channel selection dynamics in dense wi-fi networks," Communications Magazine, IEEE, vol. 53, no. 1, pp. 110-117, January 2015.

[15] S. S. Sagari, "Coexistence of LTE and WiFi heterogeneous networks via inter network coordination," in Proceedings of the 2014 Workshop on PhD Forum, New York, NY, USA, 2014, PhD forum '14, pp. 1-2, ACM.

[16] Mung Chiang, Chee Wei Tan, D.P. Palomar, D. O’Neill, and D. Julian, "Power control by geometric programming," Wireless Communications, IEEE Transactions on, vol. 6, no. 7, pp. 2640-2651, July 2007.

[17] IEEE, "Part 11: Wireless LAN medium access control (MAC) and physical layer (PHY) specifications," 2012.

[18] Kyle Jamieson, Bret Hull, Allen Miu, and Hari Balakrishnan, "Understanding the real-world performance of carrier sense," in Proceedings of the 2005 ACM SIGCOMM Workshop on Experimental Approaches to Wireless Network Design and Analysis, New York, NY, USA, 2005, E-WIND '05, pp. 52-57, ACM.
[19] G. Bianchi, "Performance analysis of the IEEE 802.11 distributed coordination function," IEEE Journal on Selected Areas in Communications, vol. 18, no. 3, pp. $535-547,2000$.

[20] Chaves F. S., Cavalcante A. M., Almeida E. P. L., Abinader F. M. Jr., Vieira R. D., Choudhury S., and Doppler K., "LTE/Wi-Fi coexistence: Challenges and mechanisms," 2013, XXXI SIMPOSIO BRASILEIRO DE TELECOMUNICACOES - SBrT2013.

[21] F.M. Abinader, E.P.L. Almeida, F.S. Chaves, A.M. Cavalcante, R.D Vieira, R.C.D. Paiva, A.M. Sobrinho, S. Choudhury, E. Tuomaala, K. Doppler, and V.A. Sousa, "Enabling the coexistence of lte and wi-fi in unlicensed bands," Communications Magazine, IEEE, vol. 52, no. 11, pp. 54-61, Nov 2014.

[22] A. Baid, S. Mathur, I. Seskar, S. Paul, A. Das, and D. Raychaudhuri, "Spectrum mri: Towards diagnosis of multi-radio interference in the unlicensed band," in Wireless Communications and Networking Conference (WCNC), 2011 IEEE, March 2011, pp. 534-539.

[23] E. Almeida, A.M. Cavalcante, R.C.D. Paiva, F.S. Chaves, F.M. Abinader, R.D. Vieira, S. Choudhury, E. Tuomaala, and K. Doppler, "Enabling LTE/WiFi coexistence by LTE blank subframe allocation," in 2013 IEEE International Conference on Communications (ICC), June 2013, pp. 5083-5088.

[24] S.K. Baghel, M.A. Ingale, and G. Goyal, "Coexistence possibilities of lte with ism technologies and gnss," in Communications (NCC), 2011 National Conference on, Jan 2011, pp. 1-5.

[25] A.M. Cavalcante, E. Almeida, R.D. Vieira, F. Chaves, R.C.D. Paiva, F. Abinader, S. Choudhury, E. Tuomaala, and K. Doppler, "Performance evaluation of LTE and Wi-Fi coexistence in unlicensed bands," in 2013 IEEE 77th Vehicular Technology Conference (VTC Spring), June 2013, pp. 1-6.

[26] Inc Qualcomm Technologies, "LTE in unlicensed spectrum: Harmonious coexistence with Wi-Fi," 2014, White paper.

[27] R. Ratasuk, M.A. Uusitalo, N. Mangalvedhe, A. Sorri, S. Iraji, C. Wijting, and A. Ghosh, "License-exempt LTE deployment in heterogeneous network," in Wireless Communication Systems (ISWCS), 2012 International Symposium on, Aug 2012, pp. 246-250.

[28] Feilu Liu, E. Bala, E. Erkip, and Rui Yang, "A framework for femtocells to access both licensed and unlicensed bands," in Modeling and Optimization in Mobile, Ad Hoc and Wireless Networks (WiOpt), 2011 International Symposium on, May 2011, pp. 407-411.

[29] T. Nihtila, V. Tykhomyrov, O. Alanen, M.A. Uusitalo, A. Sorri, M. Moisio, S. Iraji, R. Ratasuk, and N. Mangalvedhe, "System performance of LTE and IEEE 802.11 coexisting on a shared frequency band," in Wireless Communications and Networking Conference (WCNC), 2013 IEEE, April 2013, pp. 1038-1043.

[30] F.S. Chaves, E.P.L. Almeida, R.D. Vieira, A.M. Cavalcante, F.M Abinader, S. Choudhury, and K. Doppler, "LTE UL power control for the improvement of LTE/Wi-Fi coexistence," in 2013 IEEE 78th Vehicular Technology Conference (VTC Fall), Sept 2013, pp. 1-6.

[31] R.C.D. Paiva, P. Papadimitriou, and S. Choudhury, "A physical layer framework for interference analysis of LTE and Wi-Fi operating in the same band," in 2013 Asilomar Conference on Signals, Systems and Computers, Nov 2013, pp. 1204-1209.

[32] D. Raychaudhuri, I. Seskar, M. Ott, S. Ganu, K. Ramachandran, H. Kremo, R. Siracusa, H. Liu, and M. Singh, "Overview of the orbit radio grid testbed for evaluation of next-generation wireless network protocols," in Wireless Communications and Networking Conference, 2005 IEEE, March 2005, vol. 3, pp. 1664-1669 Vol. 3.

[33] Ettus Research, "Usrp b200 and b210," http://tinyurl.com/k7w6zh2.

[34] Linux Wireless ath9k, ," https://wireless.wiki.kernel.org/en/users/drivers/ath9k.

[35] "hostapd: Ieee 802.11 ap, ieee 802.1x/wpa/wpa2/eap/radius authenticator," http://w1.fi/hostapd/.

[36] Navid Nikaein, Mahesh K. Marina, Saravana Manickam, Alex Dawson, Raymond Knopp, and Christian Bonnet, "Openairinterface: A flexible platform for 5g research," SIGCOMM Comput. Commun. Rev., vol. 44, no. 5, pp. 33-38, Oct. 2014.

[37] A. Baid, M. Schapira, I. Seskar, J. Rexford, and D. Raychaudhuri, "Network cooperation for client-AP association optimization," in IEEE WiOpt, 2012, pp. $431-436$.

[38] Linux Wireless, ," https://wireless.wiki.kernel.org/en/users/documentation/iw. 
[39] Aditya Gudipati, Daniel Perry, Li Erran Li, and Sachin Katti, "Softran: Software defined radio access network," in Proceedings of the Second ACM SIGCOMM Workshop on Hot Topics in Software Defined Networking, New York, NY, USA, 2013, HotSDN '13, pp. 25-30, ACM. 